\title{
评述
}

\section{第一性原理计算在非线性光学材料领域的应用及 进展}

\author{
张兵兵, 武奎, 王颖, 杨大清
}

河北大学化学与环境科学学院, 保定 071002

*通讯作者, E-mail: zhangbb@hbu.edu.cn

收稿日期: 2021-06-02; 接受日期: 2021-08-04; 网络版发表日期: 2021-09-10

国家自然科学基金面上项目(编号: 5207021329, 21975062, 51872324)、河北省自然科学基金(编号: B2019201433, E2019201049)和河北大学高 层次引进人才项目(编号: 521000981279$)$ 资助

摘要非线性光学晶体材料需要同时满足多个性能指标, 使得发现新的非线性光学晶体成为一项充满挑战且 耗时耗力的工作. 基于第一性原理的计算材料方法可以通过预测材料的性能从大量的材料中篮选出符合性能指 标的材料. 另外, 通过研究材料的“组成-结构-性能”关系, 可以鉴定材料的非线性光学活性基团, 为新材料的合成 提供指导. 本文总结了近年来计算材料在发现新型非线性光学材料中所起到的作用和案例，介绍了目前最新发 展的非线性光学材料第一性原理高通量笁选流水线系统(FHSP-NLO). 该系统结合了密度泛函理论(DFT)计算程 序, 线性、非线性光学性质计算程序, 数据转换和提取程序, 实现了对材料性能的自动化和大批量计算. 在包括 厥酸盐、锗酸盐、氮代硅酸盐体系中取得了成功应用, 而且为寻找非线性光学材料提供了一个强大而有效的 工具.

关键词非线性光学晶体, 二次谐波产生, 第一性原理, 高通量䇻选

\section{1 引言}

自1960年Theodore Maiman发明了红宝石激光器, 激光作为 20 世纪人类最重要的发明之一, 在工业和科 研领域起到了重要作用。1961年，Franken等 ${ }^{[1]}$ 用红宝 石激光器照射石英晶体, 在出射光中检测到了波长为 入射光一半、频率为入射光两倍的新光束. 这一发现 开启了非线性光学这一新的学科分支. 非线性光学晶 体作为产生非线性光学现象的重要介质，通过二次谐 波产生、和频、差频、光参量放大、线性电光效应、
光整流效应等二阶非线性光学效应实现对激光的调 控, 是一类重要的光电功能材料, 在拓展激光器频率、 超短/超强激光脉冲、光通信、产生纠缠光子对等领 域有重要应用 ${ }^{[2]}$. 寻找性能优异的非线性光学晶体材 料是一项充满挑战但意义重大的工作 ${ }^{[3 \sim 10]}$.

按照工作波段, 非线性光学晶体可分为四个波段, 分别是深紫外(DUV， < $200 \mathrm{~nm})$ 、紫外波段(UV, 200 400 nm), 可见/近红外波段(vis-NIR，0.4 3 $\mu \mathrm{m}$ ), 以及中/远红外波段(M-F-IR，包括3 5和8 13 $\mu \mathrm{m}$ 大气 透明窗口). 经过半个多世纪的发展, 科学家发现了一

引用格式: Zhang B, Wu K, Wang Y, Yang D. The applications and advances of the first principles calculation in nonlinear optical materials. Sci Sin Chim, 2021, 51: 1137-1149, doi: 10.1360/SSC-2021-0137 
批可以应用在可见/近红外和紫外波段的非线性光学 晶体, 包括 $\mathrm{KH}_{2} \mathrm{PO}_{4}(\mathrm{KDP})^{[11]} 、 \mathrm{LiNbO}_{3}(\mathrm{LN})^{[12]} 、 \mathrm{KTiO}-$ $\mathrm{PO}_{4}(\mathrm{KTP})^{[13]} 、 \beta-\mathrm{BaB}_{2} \mathrm{O}_{4}(\mathrm{BBO})^{[14]}, \mathrm{LiB}_{3} \mathrm{O}_{5}$ $(\mathrm{LBO})^{[15]} 、 \mathrm{CsB}_{3} \mathrm{O}_{5}(\mathrm{CBO})^{[16]}, \mathrm{CsLiB}_{6} \mathrm{O}_{10}(\mathrm{CLBO})^{[17]}$ 等. 在深紫外波段, 目前实验上能通过直接倍频输出 短于 $200 \mathrm{~nm}$ 激光的只有 $\mathrm{KBe}_{2} \mathrm{BO}_{3} \mathrm{~F}_{2}(\mathrm{KBBF})^{[14]}$ 晶体. 但是该晶体严重的层状生长习性阻碍了大尺寸单晶的 生长, 从而影响了该晶体的大规模应用. 在中远红外波 段，AgGaSe2、AgGaS2、ZnGeP2 是最具代表性的实 用化晶体 ${ }^{[18]}$. 然而, $\mathrm{AgGaSe}_{2}$ 和 $\mathrm{AgGaS}_{2}$ 存在激光损伤 阈值(LDT)低( $20 \mathrm{MW} / \mathrm{cm}^{2}$ 量级, $1064 \mathrm{~nm}$ ，脉宽 $\tau_{\mathrm{p}}=20 \mathrm{~ns}$ ) 等问题. $\mathrm{ZnGeP}_{2}$ 带隙较窄, 对 $1 \mu \mathrm{m}$ 的抽运光 双光子吸收严重; 非本征缺陷引起 $\mathrm{ZnGeP}_{2}$ 晶体的光吸 收和光散射, 造成晶体在 $2 \mu \mathrm{m}$ 处透过率低. 针对目前的 现状, 非线性光学材料的研究前沿聚焦于深紫外波段 和中远红外波段.

非线性光学晶体需要同时满足带隙、双折射、倍 频系数等多个性能指标 ${ }^{[14]}$ ，而这些性能之间有一些是 相互制约的. 普遍地，倍频系数随着带隙的增加而明 显降低, 双折射也与带隙呈负相关. 因此, 只有非常少 的材料可以同时满足多重性能指标. 除了这些材料的 本征物理性能之外，还要求材料容易生长大尺寸、高 纯度的单晶, 容易加工成器件, 并且在工作状态下物 化性能稳定. 这些要求进一步增加了获得可实用化非 线性光学晶体材料的难度.

发现新材料是一个耗时耗力的过程. 新材料的合 成需要大量的反复试验才能确定最佳的合成条件和反 应物配比，并且某些化学反应需要几天甚至几周的时 间才能完成. 大多数化学组成简单，容易合成的化合 物已被前人报道，发现新材料的方式倾向于选择更复 杂的组分，采取更苛刻的反应条件，这致使发现新化 合物的难度呈逐渐增大的趋势，同时也增加了晶体生 长的难度.

为了加速新材料的发现，科学家希望找到材料的 “组成-结构-性能”之间的关系，知道材料中哪些元素 组成和结构基团对性能起到关键作用，为新材料的发 现与合成提供理论指导. 20 世纪 80 年代，陈创天院 $士^{[19]}$ 提出了“阴离子基团理论”，基于该理论，开发出 了计算机辅助材料设计系统(CAMDS). 该系统在硼酸 盐体系获得了巨大的成功，帮助他们发现了著名的中 国牌晶体 $\mathrm{LBO} 、 \mathrm{CBO}$ 和 $\mathrm{KBBF}$ 等。这也使得我国在这
一领域处于全球领先的位置. 随后，陈创天院士与李 明宪等 ${ }^{[20]}$ 合作引入第一性原理方法，合作开发了倍频 系数计算程序, 实空间原子切割、倍频密度等工具 ${ }^{[20]}$. 这些工具在计算材料倍频系数, 解释倍频效应来源, 定 位功能基团的工作中起到了重要作用. 此外, 林哲帅课 题组发展了带隙预测模型modified-bond-valence-sum $(\mathrm{MBVS})^{[21]}$ 和解释倍频来源的柔度因子模型 ${ }^{[22]}$. 潘世 烈和杨志华课题组提出了双折射计算模型REDA ${ }^{[23]}$ 、 材料模块导向设计策略 ${ }^{[24,25]}$. Rondinelli课题组 ${ }^{[26]}$ 开发 了 specific acentric-mode displacements (SAMD)方法解 释倍频效应的来源.

除了用来解释非线性光学效应起源, 第一性原理 方法也可以用来预测新材料和从已知的材料中篮选 非线性光学晶体材料. 随着高性能计算资源的发展和 计算准确性的提高, 基于第一性原理预测和篎选新功 能材料正在成为加快材料研发的强大工具, 受到了越 来越多的关注. 计算辅助可以篮选和减少需要进行实 验的总数，而不用对每个材料都进行实验合成并测 试. 基于密度泛函理论(DFT)的高通量篮选方法已成 功应用于材料科学领域，如锂离子电池、热电材 料、超导材料. 在非线性光学材料领域, 虽然有多个 通过理论预测和计算管出优异材料的例子, 但是处理 的材料数目有限. 人类合成并测试了数量巨大的无机 晶体材料，有 20 余万个结构收录在无机晶体数据库 (ICSD)中。这其中很多材料没有被有效地挖掘和开 发, 是获得新材料的一座宝库. 受限于研究方向和实 验条件, 很多具有非中心对称结构材料的非线性光学 效应没有被有效发掘. 由于无机晶体材料数目巨大, 如果没有高效的搜寻策略和可靠的理论指导，从中 寻找性能优异的非线性光学材料犹如大海捞针, 面 临巨大的挑战. 针对这一现状，本课题组开发了非线 性光学材料第一性原理高通量篎选流水线系统 $(\text { FHSP-NLO })^{[27]}$, 该系统结合了DFT计算程序, 非线性 光学性质计算程序, 以及数据转换和提取程序. 该系 统可以自动运行，一次性计算和分析成百上千个材 料. 这可以用于在无机晶体结构数据库中搜索有潜 力的非线性光学材料.

本文综述了第一性原理方法在发现非线性光学晶 体材料过程中发挥的重要作用, 尤其是在已知材料中 笁选和鉴定新型结构基团的工作. 着重介绍了非线性 光学材料FHSP-NLO流水线系统的开发及其应用. 


\section{2 第一性原理方法辅助发现新型非线性光 学材料和功能基团}

\section{1 深紫外非线性光学晶体}

\subsection{1 氟代嗍酸盐非线性光学晶体}

深紫外激光在微加工、激光光刻、高密度存储、 超高分辨率光电子能谱仪等方向具有重要的应用 ${ }^{[28]}$. 目前, 只有自由电子激光和 $\mathrm{KrF}$ 等准分子激光器可以 产生商业化应用的深紫外激光. 固体激光器因其体积 小, 出光稳定, 能量转化率高, 克服了已有深紫外激光 器的缺点, 但是高功率全固态激光器的输出波长集中 在红外波段, 必须利用非线性光学(NLO)频率转换材 料才可以获得深紫外激光 ${ }^{[29,30]}$. 近年来, 随着激光技 术的发展，非线性光学晶体材料及其相关机制成为当 前材料、物理科学的前沿课题之一.

嶰酸盐晶体在产生短波长激光光源上具有明显优 势，为了获得可以达到深紫外透过的硼酸盐非线性光 学材料, 需要消除氧原子的悬挂键. 其中一种策略是 使材料形成BO基团组成的三维网状结构, 如LBO. 但 是这种三维网络结构的光学各向异性较低, 无法获得 大的双折射来实现深紫外的相位匹配. 另一种策略是 引入可以和氧形成较强共价键的阳离子，如 $\mathrm{Be} 、 \mathrm{Al}$ 等 来消除平行排列 $\mathrm{BO}_{3}$ 基团上 $\mathrm{O}$ 的悬挂键, 典型的材料如 $\mathrm{KBBF}$ 和 $\mathrm{SBBO}^{[31]}$. 但是这种策略导致材料层间结合力 较弱, 不利于生长大尺寸晶体(KBBF)或者产生堆垛层 错(SBBO). 科学家一直在寻找新的材料设计策略来 克服嗍酸盐中存在的上述矛盾。2009 2012年期间， Jansen课题组 ${ }^{[32 ~ 34]}$ 合成了一系列氟代嗍酸锂盐用于离 子导电研究. 随后, 张兵兵等 ${ }^{[35]}$ 在对这一系列材料进行 第一性原理计算和结构性能关系研究后发现, $\mathrm{Li}_{2} \mathrm{~B}_{6} \mathrm{O}_{9}$ $\mathrm{F}_{2}$ 可以同时实现大的带隙和双折射, 同时避免了层状结 构. 据此提出了一种可以在增大材料双折射的同时避 免层状生长习性并且截止边达到深紫外的材料设计策 略. 指出了一类新的应用于深紫外波段的功能基团 $\left(\mathrm{BO}_{3} \mathrm{~F}\right)^{4-} 、\left(\mathrm{BO}_{2} \mathrm{~F}_{2}\right)^{3-}$ 和 $\left(\mathrm{BOF}_{3}\right)^{2-}$ (通式为 $\left(\mathrm{BO}_{x} \mathrm{~F}_{4-x}\right)^{(x+1)-}$, $x=1 、 2 、 3)$. 如图1所示，与 $\mathrm{O}$ 原子不同， $\mathrm{F}$ 原子在结构 中只与一个 $\mathrm{B}$ 原子相连, 对 $\mathrm{B}-\mathrm{O}$ 网络结构具有 “剪切作 用”. 可以打破不利于产生大双折射的三维B-O网络结 构, 增加结构的多样性, 有利于获得大双折射的结构. 该策略同时避免了端氧的形成, 有利于深紫外透过. 第 一性原理计算表明， $[\mathrm{BOF}]$ 基团拥有比 $\left(\mathrm{BO}_{4}\right)^{5-}$ 大的超

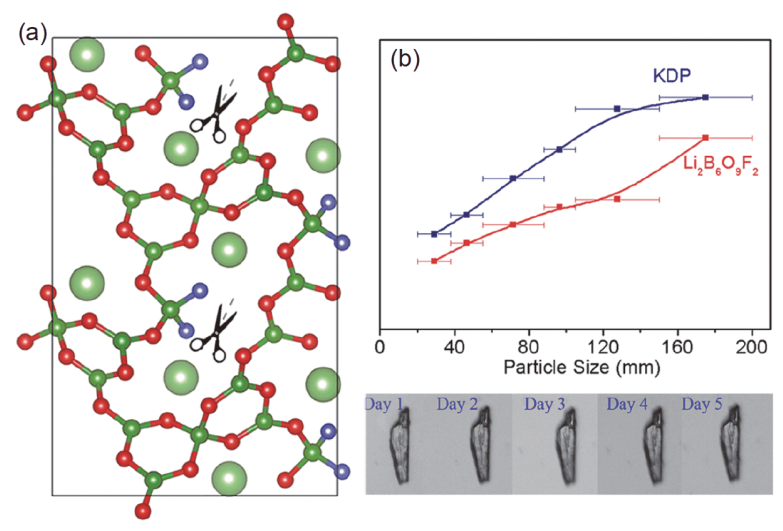

图 1 (a) $\mathrm{Li}_{2} \mathrm{~B}_{6} \mathrm{O}_{9} \mathrm{~F}_{2}$ 的晶体结构及 $\left(\mathrm{BO}_{2} \mathrm{~F}_{2}\right)^{3-}$ 基团的“剪切”作 用; (b) 粉末倍频效应及晶体照片 (晶体照片表明其在空气中 无明显变化 $)^{[35]}$ (网络版彩图)

Figure 1 (a) The crystal structure of $\mathrm{Li}_{2} \mathrm{~B}_{6} \mathrm{O}_{9} \mathrm{~F}_{2}$, in which $\left(\mathrm{BO}_{2} \mathrm{~F}_{2}\right)^{3-}$ cut off $\mathrm{B}-\mathrm{O}$ framework without terminal oxygens; (b) the powder SHG response and the crystal micrograph (The crystal micrographs show that the crystal is stable in air) [35] (color online).

极化率和极化率各向异性，拥有大的带隙. 实验测试 和理论计算表明， $\mathrm{Li}_{2} \mathrm{~B}_{6} \mathrm{O}_{9} \mathrm{~F}_{2}$ 具有合适的倍频效应 $(0.9 \times \mathrm{KDP})$ 和大的双折射 $(0.07)$, 同时因其开放的网络 结构避免了层状生长习性, 是一例潜在的深紫外非线 性光学材料. 该材料可以突破深紫外非线性光学材料 各性能指标之间的矛盾限制，打破深紫外瓶颈。深入 的理论分析表明, $\left(\mathrm{BO}_{x} \mathrm{~F}_{4-x}\right)^{(x+1)-}$ 功能基团的引入一方 面可以保持嗍酸盐在透过方面的优点，同时可以增加 材料的光学各向异性, 提升材料的倍频系数和双折射.

之后，一系列氟代硣酸盐深紫外非线性光学晶体 材料被发现和报道, 包括 $\mathrm{NH}_{4} \mathrm{~B}_{4} \mathrm{O}_{6} \mathrm{~F}^{[36]} 、 \mathrm{NaB}_{4} \mathrm{O}_{6} \mathrm{~F}^{[37]}$ 、 $\mathrm{RbB}_{4} \mathrm{O}_{6} \mathrm{~F}^{[38]}, \mathrm{CsKB}_{8} \mathrm{O}_{12} \mathrm{~F}_{2}{ }^{[38]} 、 \mathrm{CsB}_{4} \mathrm{O}_{6} \mathrm{~F}^{[39]} 、 \mathrm{CaB}_{5} \mathrm{O}_{7^{-}}$ $\mathrm{F}_{3}{ }^{[40,41]} 、 \mathrm{SrB}_{5} \mathrm{O}_{7} \mathrm{~F}_{3}{ }^{[40,42]} 、 \mathrm{MgB}_{5} \mathrm{O}_{7} \mathrm{~F}_{3}{ }^{[43]}$. 根据计算的折 射率, 这些材料均可以实现小于 $200 \mathrm{~nm}$ 的相位匹配. 科研人员对其中一部分材料进行了晶体生长，没有发 现明显的层状生长习性.

\subsection{2 氟代磷酸盐非线性光学晶体}

磷酸盐具有短的紫外吸收截止边，且可以获得较 大的非线性效应，是研究深紫外非线性光学材料的潜 在体系. 但是由于磷酸盐中 $\mathrm{PO}_{4}$ 基团光学各向异性非 常小，很难获得较大的双折射以满足角度相位匹配. 张兵兵等 ${ }^{[44]}$ 通过材料基因方法提出了一类新的紫外/ 深紫外非线性光学材料研究体系——氟代磷酸盐, 拓 展了深紫外非线性光学材料的研究方向, 提出了通过 
$\left(\mathrm{PO}_{3} \mathrm{~F}\right)^{2-}$ 和 $\left(\mathrm{PO}_{2} \mathrm{~F}_{2}\right)$ 基团替换 $\left(\mathrm{PO}_{4}\right)^{3-}$ 基团来提升磷酸 盐晶体的双折射. 第一性原理计算表明, $\left(\mathrm{PO}_{3} \mathrm{~F}\right)^{2-}$ 和 $\left(\mathrm{PO}_{2} \mathrm{~F}_{2}\right)$ 基团具有非常大的最高已占轨道-最低未占轨 道(HOMO-LUMO)能隙、较大的极化率各向异性和超 极化率, 可以增强晶体的双折射, 同时产生大的二阶倍 频效应和短截止边. 在ICSD中搜索并应用第一性原理 方法计算了含有这些基团材料的性能. 如图2所示, 氟 代磷酸盐的双折射比磷酸盐显著提高. 根据计算结果, 篮选出了一例紫外非线性光学材料: $\left(\mathrm{NH}_{4}\right)_{2} \mathrm{PO}_{3} \mathrm{~F}$, 通过 溶剂蒸发法生长了厘米级大小的单晶, 测试发现其满 足 $266 \mathrm{~nm}$ 倍频光相位匹配. 更重要的是，氟代磷酸盐 具有比硼酸盐更小的折射率色散，降低了其在深紫外 波段实现相位匹配对双折射的要求。该工作提出的氟 代磷酸盐拓展了该领域的研究方向，并验证了通过第 一性原理高通量篮选可以高效地获得光功能材料. 之 后，吴立明课题组 ${ }^{[45]}$ 报道了单氟代磷酸作为深紫外非 线性光学材料的优势体系, 篮选出了三例单氟代磷酸 盐, 即 $\left(\mathrm{NH}_{4}\right)_{2} \mathrm{PO}_{3} \mathrm{~F} 、\left(\mathrm{C}\left(\mathrm{NH}_{2}\right)_{3}\right)_{2} \mathrm{PO}_{3} \mathrm{~F} 、 \mathrm{NaNH}_{4} \mathrm{PO}_{3} \mathrm{~F} \cdot \mathrm{H}_{2}$ O. 2019 年, 吴立明、陈玲课题组 ${ }^{[46]}$ 在引入高极化各向 异性的基团 $\mathrm{PO}_{3} \mathrm{~F}^{2-}$ 的基础上又提出将氢键引入晶体 中, 用氢键的束缚力使高极化各向异性的 $\left(\mathrm{PO}_{3} \mathrm{~F}\right)^{2-}$ 离 子整齐排列，显著提高了磷酸盐的各向异性，获得了 $\mathrm{NaNH}_{4} \mathrm{PO}_{3} \mathrm{~F} \cdot \mathrm{H}_{2} \mathrm{O}$ 晶体, 使双折射从 0.03 提升到了 0.053 , 为氟代磷酸盐体系中最大值.

\subsection{3 聚二氟磷腈 $\left(\mathrm{PNF}_{2}\right)_{n}$ 非线性光学晶体}

除了多面体和平面基团，一维的链可能是另一种 深紫外NLO材料的功能基团. 2019年，康雷等 ${ }^{[47]}$ 提出 以 $-\mathrm{P}=\mathrm{N}-\mathrm{P}=\mathrm{N}-\mathrm{P}=\mathrm{N}-$ 为一维骨架的磷腈聚合物是一类 潜在的深紫外非线性光学材料基团. 根据 $\mathrm{P}$ 上配位原子 的不同, 磷腈阴离子基团的构成单元可分为 $\left(\mathrm{PN}_{4}\right)^{7-}$ 、 $\left(\mathrm{PN}_{2} \mathrm{O}_{2}\right)^{5-}$ 和 $\left(\mathrm{PN}_{2} \mathrm{XY}\right)^{3-}(\mathrm{X}, \mathrm{Y}=\mathrm{F}, \mathrm{Cl}, \mathrm{Br})$. 他们用第一 性原理方法计算了 ICSD数据库中不同类型的含磷腈 阴离子基团的化合物. 通过计算发现, 由 $\left(\mathrm{PNF}_{2}\right)_{n}$ 一维 链组成的 $\mathrm{PNF}_{2}$ 具有优异的综合性能(图3), 带隙 $E_{\mathrm{g}} \approx$ $8.8 \mathrm{eV}$, 非线性光学系数 $d_{33} \approx 0.86 \mathrm{pm} / \mathrm{V}$, 双折射 $\Delta n \approx 0.16$. 根据计算得到的折射率预测的最短相位匹 配波长为 $142 \mathrm{~nm}$, 短于 $\mathrm{KBBF}(161 \mathrm{~nm})$.

\subsection{4 氧基 $(\mathrm{CN})$ 和羰基(CO)非线性光学晶体}

$\mathrm{C}$ 和 $\mathrm{N}$ 通过三键结合成的基团称为氰基. 康雷等 ${ }^{[48]}$

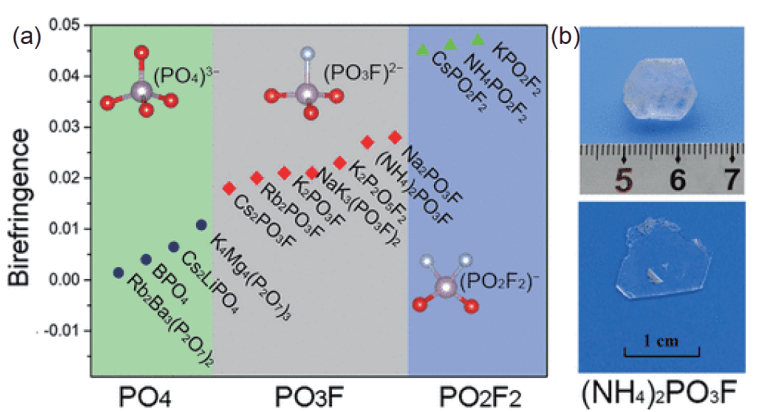

图 2 (a) 含有 $\left(\mathrm{PO}_{3} \mathrm{~F}\right)^{2-}$ 和 $\left(\mathrm{PO}_{2} \mathrm{~F}_{2}\right)$ 基团的无机晶体材料的双 折射; (b) 生长的 $\left(\mathrm{NH}_{4}\right)_{2} \mathrm{PO}_{3} \mathrm{~F}$ 单晶 ${ }^{[44]}$ (网络版彩图)

Figure 2 (a) The calculated birefringence of inorganic crystals containing $\left(\mathrm{PO}_{3} \mathrm{~F}\right)^{2-}$ and $\left(\mathrm{PO}_{2} \mathrm{~F}_{2}\right)^{-}$anionic groups. (b) The single crystal of $\left(\mathrm{NH}_{4}\right)_{2} \mathrm{PO}_{3} \mathrm{~F}$ [44] (color online).

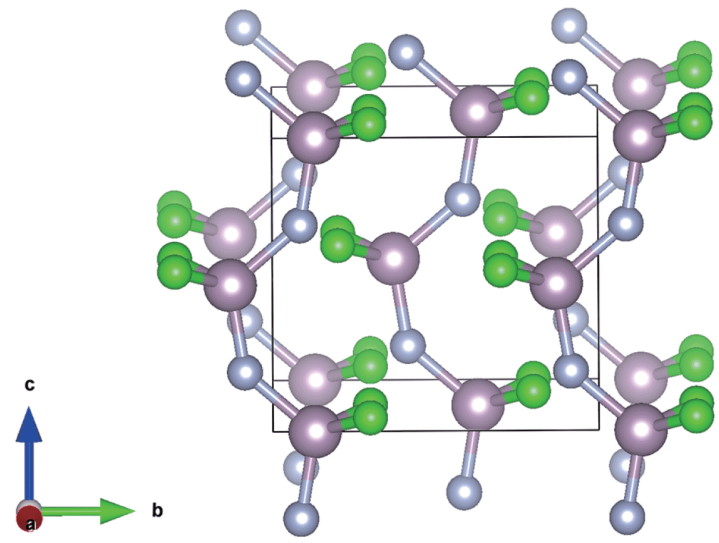

图 $3 \mathrm{PNF}_{2}$ 的晶体结构 (网络版彩图)

Figure 3 The crystal structure of $\mathrm{PNF}_{2}$ (color online).

调研了若干种含氰基的化合物，包括金属氰化物、卤 素氧化物和硫属氰化物。通过第一性原理计算表明, 氧基化合物可以导致大的倍频系数，同时具有很大的 双折射(均大于 0.1 , 有些甚至大于 0.5 ). 从中篮选出了 $\beta-\mathrm{KCN}$, 可实现深紫外输出, 最短相位匹配波长达到 $193.7 \mathrm{~nm}$. CNI可是实现 $266 \mathrm{~nm}$ 相位匹配, 具有大的倍 频系数和双折射. $\mathrm{CNBr}$ 和 $\mathrm{CNCl}$ 可实现 $177.3 \mathrm{~nm}$ 深紫外 输出.

此外, 该课题组 ${ }^{[49]}$ 还研究了含有 $\mathrm{C}-\mathrm{O}$ 基的化合物, 第一性原理计算表明, $\left(\mathrm{Cl}_{2} \mathrm{~B}\right)_{3} \mathrm{BCO}$ 和 $\mathrm{SCO}$ 具有大的双 折射和倍频系数, 其中 SCO 有望实现 $193.7 \mathrm{~nm}$ 的深紫 外相位匹配. 但遗憾的是, 受限于材料合成方法和材 料的稳定性, 磷腈聚合物、氭基和羰基化合物均没有 做进一步的实验验证，也没有在这些体系发现新的非 
线性光学材料.

\section{2 紫外非线性光学晶体}

\subsection{1 氧尿酸盐非线性光学晶体}

2013年开始, Meyer等 ${ }^{[50 \sim 53]}$ 合成了一系列含有 $\left(\mathrm{C}_{3} \mathrm{~N}_{3} \mathrm{O}_{3}\right)^{3-}$ 基团的氰尿酸盐晶体, 发现部分材料具有 二阶非线性光学效应，包括 $\mathrm{Ca}_{3}\left(\mathrm{C}_{3} \mathrm{~N}_{3} \mathrm{O}_{3}\right)_{2} 、 \alpha-\mathrm{Sr}_{3}-\left(\mathrm{C}_{3} \mathrm{~N}_{3}\right.$ $\left.\mathrm{O}_{3}\right)_{2} 、 \beta-\mathrm{Sr}_{3}\left(\mathrm{C}_{3} \mathrm{~N}_{3} \mathrm{O}_{3}\right)_{2}$ 和 $\mathrm{Eu}_{3}\left(\mathrm{C}_{3} \mathrm{~N}_{3} \mathrm{O}_{3}\right)_{2} .2017$ 年，梁飞 等 ${ }^{[54]}$ 采用第一性原理方法对 $\mathrm{Ca}_{3}-\left(\mathrm{C}_{3} \mathrm{~N}_{3} \mathrm{O}_{3}\right)_{2}$ 和 $\beta-\mathrm{Sr}_{3}$ $\left(\mathrm{C}_{3} \mathrm{~N}_{3} \mathrm{O}_{3}\right)_{2}$ 进行了详细的理论研究. $\left(\mathrm{C}_{3} \mathrm{~N}_{3} \mathrm{O}_{3}\right)^{3-}$ 基团具 有与 $\left(\mathrm{B}_{3} \mathrm{O}_{6}\right)^{3-}$ 类似的 $\pi$-共轭电子结构, 但是电子分布 有很大差别. $\mathrm{Sr}_{3}\left(\mathrm{C}_{3} \mathrm{~N}_{3} \mathrm{O}_{3}\right)_{2}$ 的计算非线性光学系数 $d_{22}$ 为 $3.93 \mathrm{pm} / \mathrm{V}$, 实空间原子切割表明 $\left(\mathrm{C}_{3} \mathrm{~N}_{3} \mathrm{O}_{3}\right)^{3-}$ 基团对 倍频的贡献超过 $90 \%$, 占绝对主导作用. 2019年, 该课 题 ${ }^{[55]}$ 用第一性原理方法在晶体开放数据库(COD)中 篮选出来了 $\mathrm{LiCl} \cdot\left(\mathrm{H}_{3} \mathrm{C}_{3} \mathrm{~N}_{3} \mathrm{O}_{3}\right)$ 晶体. 计算表明, 该晶体 紫外截止边为 $215 \mathrm{~nm}$, 双折射 0.28 , 倍频系数为 $4.15 \mathrm{pm} / \mathrm{V}$.

在实验领域, 夏明军等 ${ }^{[56]}$ 在2018年合成了一种新 的氰尿酸盐 $\mathrm{K}_{6} \mathrm{Cd}_{3}-\left(\mathrm{C}_{3} \mathrm{~N}_{3} \mathrm{O}_{3}\right)_{4}$, 其实验带隙为 $5.23 \mathrm{eV}$. 计算的非线性光学系数 $d_{14}=1.07 \mathrm{pm} / \mathrm{V}$. 随后, 该课题 组 ${ }^{[57]}$ 合成了一系列氰尿酸盐, 其表现出丰富的结构多 样性和优良的非线性光学性能.

叶宁课题组 ${ }^{[58]}$ 成功合成出了具有非线性光学效应 的 $\mathrm{ABHC}_{3} \mathrm{~N}_{3} \mathrm{O}_{3} \cdot x \mathrm{H}_{2} \mathrm{O}(\mathrm{AB}=\mathrm{KLi} 、 \mathrm{RbLi} 、 \mathrm{RbNa} 、 \mathrm{CsNa})$ 系列氧尿酸盐晶体, 并进一步以水溶液法得到了 $\mathrm{KLiHC}_{3} \mathrm{~N}_{3} \mathrm{O}_{3} \cdot 2 \mathrm{H}_{2} \mathrm{O}$ (KLHCY) 厘米级大单晶. $\mathrm{KLHCY}$ 晶体的一类相位匹配最短波长能达到 $246 \mathrm{~nm}$, 激光损伤 阈值达到 $4.76 \mathrm{GW} / \mathrm{cm}^{2}$, 是极具应用前景的新一代紫外 非线性光学晶体.

吴立明、陈玲课题组 ${ }^{[59]}$ 以 $\left(\mathrm{C}_{3} \mathrm{~N}_{3} \mathrm{O}_{3}\right)^{3-}$ 基团为构筑 单元, 设计并合成了 $\left(\mathrm{C}_{3} \mathrm{~N}_{3} \mathrm{O}_{3}\right)^{3-}$ 环完美共面排列的氧 尿酸盐 $\mathrm{Rb}_{2} \mathrm{HC}_{3} \mathrm{~N}_{3} \mathrm{O}_{3}$, 其双折射达到了当时氰尿酸盐家 族中最大值 $(\Delta n=0.4)$. 还设计合成了 $\mathrm{LiRbHC}_{3} \mathrm{~N}_{3} \mathrm{O}_{3} \cdot 2 \mathrm{H}_{2}$ $\mathrm{O} 、 \mathrm{NaRb}_{0 \cdot 86} \mathrm{Cs}_{0 \cdot 14} \mathrm{HC}_{3} \mathrm{~N}_{3} \mathrm{O}_{3} \cdot 2 \mathrm{H}_{2} \mathrm{O}$ 和 $\mathrm{NaRb}_{3}\left(\mathrm{H}_{2} \mathrm{C}_{3} \mathrm{~N}_{3} \mathrm{O}_{3}\right)_{4}$ $\cdot 3 \mathrm{H}_{2} \mathrm{O}$, 其二次谐波产生 $(\mathrm{SHG})$ 分别为 $2.7 、 3$ 和 0.4 倍 KDP.

自从氭尿酸盐体系受到关注以来，科学家合成了 数目众多的新材料, 取得了蓬勃发展 ${ }^{[58]}$. 截至目前, 共 合成了 85 种氰尿酸盐晶体材料, 其中 15 种具有非中心 对称结构.

\subsection{2 含 $\pi$-共轭 $\left[\mathrm{C}\left(\mathrm{NH}_{2}\right)_{3}\right]^{+}$阳离子基团的非线性光 学材料}

2020 年罗敏等 ${ }^{[60]}$ 合成了一例含胍基 $\left[\mathrm{C}\left(\mathrm{NH}_{2}\right)_{3}\right]^{+}$非 线性光学材料 $\mathrm{C}\left(\mathrm{NH}_{2}\right)_{3} \mathrm{SO}_{3} \mathrm{~F}$. 该材料具有 5 倍 $\mathrm{KDP}$ 的倍 频效应, 紫外截止边为 $200 \mathrm{~nm}$, 计算的 $1064 \mathrm{~nm}$ 处双折 射为 0.133 . 理论计算表明, 胍基对倍频系数的贡献起 主导作用 $(81 \%) .2021$ 年, 潘世烈和杨志华课题组 ${ }^{[61]}$ 在 含有胍基的中心对称材料中用第一性原理方法篮选出 了若干光学双折射材料, 其中 $\left[\mathrm{C}\left(\mathrm{NH}_{2}\right)_{3}\right]-\mathrm{HC}_{2} \mathrm{O}_{4} \cdot \mathrm{H}_{2} \mathrm{O}$ 具 有超大的双折射 $\Delta n=0.371 @ 532 \mathrm{~nm}$. 理论计算表明, $\left[\mathrm{C}\left(\mathrm{NH}_{2}\right)_{3}\right]^{+}$是双折射的主要贡献者. 叶宁课题组合成 了 $\mathrm{C}\left(\mathrm{NH}_{2}\right)_{3} \mathrm{ClO}_{4}{ }^{[62]}$ 和 $\left[\mathrm{C}\left(\mathrm{NH}_{2}\right)_{3}\right]_{3} \mathrm{PO}_{4} \cdot 2 \mathrm{H}_{2} \mathrm{O}^{[63]}$ 晶体, 测试 表明, 两种晶体的紫外截止边分别为 200 和 $250 \mathrm{~nm}$, 倍 频效应为 3 倍和 1.5 倍KDP，同时具有较大的双折射(分 别为0.076@1064 nm和0.055@546.1 nm). 吴超等 ${ }^{[64]}$ 生 长了已知结构的 $\left[\mathrm{C}\left(\mathrm{NH}_{2}\right)_{3}\right]_{6}\left(\mathrm{PO}_{4}\right)_{2} \cdot 3 \mathrm{H}_{2} \mathrm{O}$ 晶体, 测试表 明, 该晶体带隙大于 $6 \mathrm{eV}$, 倍频效应为 $3.8 \times \mathrm{KDP} @$ $1064 \mathrm{~nm}$, 同时具有较大的双折射 $(0.078 @ 546 \mathrm{~nm})$, 最 短相位匹配波长可以到 $250 \mathrm{~nm}$.

\section{3 红外非线性光学晶体}

\subsection{1 金属硫代磷酸盐非线性光学晶体}

作为潜在的红外非线性光学材料, 金属硫代磷酸 盐被研究的很少. 2015年, 康雷等 ${ }^{[65]}$ 用第一性原理计 算方法评估了金属硫代磷酸盐的非线性光学性能, 计 算了 23 个非中心对称的化合物. 按照结构特点和阳离 子种类分类讨论了这些材料. 和当时已知的红外非线 性光学材料对比发现, 金属硫代磷酸盐具有相当甚至 更优异的性能. 其中有 11 种晶体的带隙大于 $3 \mathrm{eV}$, 倍频 系数大于 10 倍 $\mathrm{KDP}$ (图4). 其中 $\mathrm{LiZnPS}_{4}$ 晶体同时具有 大的带隙和强的倍频效应, 分别是 $3.79 \mathrm{eV}$ 和 27 倍KDP. 有可能是该体系中最好的中红外非线性光学材料. 随 后挑选了 $\mathrm{Zn}_{3}\left(\mathrm{PS}_{4}\right)_{2}$ 和 $\mathrm{LiZnPS}_{4}$ 做实验合成, 带隙和倍频 系数的测试和计算值一致. 该工作对几乎所有的金属 硫代磷酸盐进行了计算和笁选，不止促进了新材料的 发现, 而且对这一系列材料提供了一种全面的组成-结 构-性能关系分析.

\subsection{2 金属硫代嗍酸盐非线性光学材料的篮选}

硫代硼酸盐(thioborates)被作为红外非线性光学 


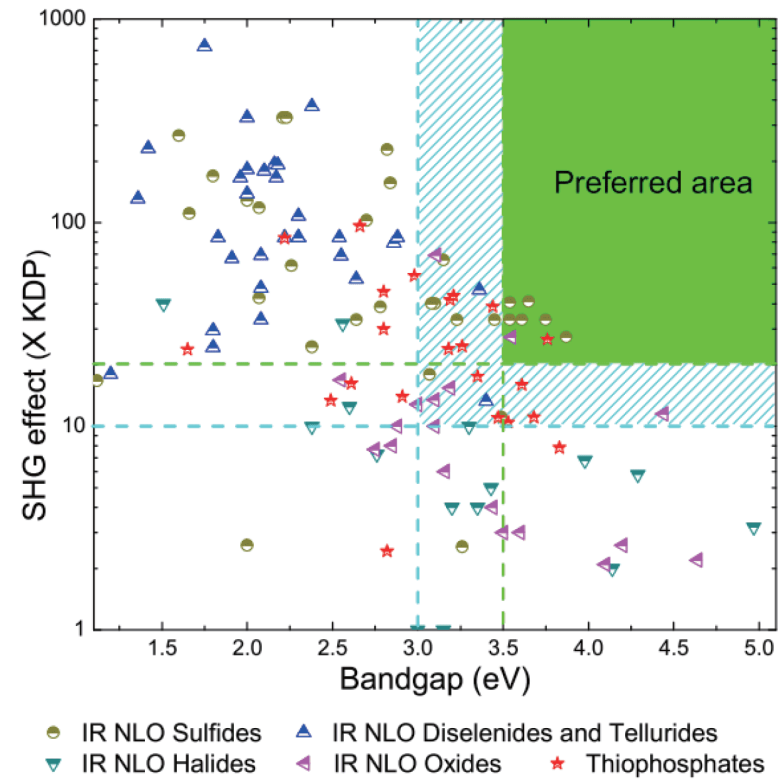

图 4 经典红外非线性光学材料的能带 $E_{\mathrm{g}}$ 和倍频系数 $d_{i j}$ 分 布. 蓝色和绿色区域分别标示 $E_{\mathrm{g}}>3.0 \mathrm{eV}$ 和 $d_{i j}>10 \times \mathrm{KDP}$ 以及 $E_{\mathrm{g}}>3.5 \mathrm{eV}$ 和 $d_{i j}>20 \times \mathrm{KDP}$ 的区域 ${ }^{[65]}$ (网络版彩图)

Figure 4 The distribution of $E_{\mathrm{g}}$ with respect to $d_{i j}$ for numerous typical IR NLO crystals. The blue and green shaded regions represent the elementary $\left(E_{\mathrm{g}}>3.0 \mathrm{eV}\right.$ and $\left.d_{i j}>10 \times \mathrm{KDP}\right)$ and preferred $\left(E_{\mathrm{g}}>3.5 \mathrm{eV}\right.$ and $\left.d_{i j}>20 \times \mathrm{KDP}\right)$ conditions, respectively, for mid-IR NLO performance [65] (color online).

材料被研究的不多. 潘世烈和杨志华课题组 ${ }^{[66]}$ 对该体 系进行了第一性原理计算研究. 计算了三个非中心对 称的金属硫代硼酸盐 $\mathrm{BaB}_{2} \mathrm{~S}_{4} 、 \mathrm{LiSrB}_{3} \mathrm{~S}_{6}$ 和 $\mathrm{LiBaB}_{3} \mathrm{~S}_{6}$. 其中 $\mathrm{BaB}_{2} \mathrm{~S}_{4}$ 倍频系数最大, 达到 0.5 倍的 $\mathrm{AgGaS}_{2}$, 带隙 $3.63 \mathrm{eV}$. 随后合成了 $\mathrm{BaB}_{2} \mathrm{~S}_{4}$ 单晶, 测试表明, 该晶体性 能优异, 带隙 $3.55 \mathrm{eV}$, 倍频效应为 $0.7 \times \mathrm{AgGaS}_{2}$, 激光损 伤阈值为8倍 $\mathrm{AgGaS}_{2}$.

\subsection{3 卤素硫属化合物非线性光学材料的篮选}

与传统硫属化物相比, 卤素硫属化物在中红外非 线性光学领域中的开发和探索尚未得到系统的研究和 关注. 潘世烈和杨志华课题组 ${ }^{[67]}$ 对 45 个具有非中心对 称结构的卤素硫属化合物进行了计算篮选(图5), 并对 其组成-结构-性能关系进行了总结和研究. 他们按所 含金属阳离子种类将该系列化合物分成含主族元素金 属和过渡族金属阳离子两大类. 根据计算结果, 笁选了 3 例性能突出的材料, 分别是 $\mathrm{CuHgSI} 、\left(\mathrm{~S}_{4} \mathrm{~N}_{4}\right)_{2} \mathrm{SnCl}_{4}$ 、 $\mathrm{Ag}_{5}\left(\mathrm{PS}_{4}\right) \mathrm{Cl}_{2}$ ．这三个材料计算的HSE带隙均大于 $2 \mathrm{eV}$, 倍频系数介于 $6.6 ~ 29 \mathrm{pm} / \mathrm{V}$ 之间. 此外, 他们还以已知
材料为模板，采用原子替代的方法设计了若干虚拟结 构，计算表明新设计的结构其性能均有所提升. 该工 作表明，卤素硫属化合物在中红外NLO材料中具有潜 在的应用价值. 将具有大电负性的卤素原子引入硫属 化物中会增大带隙, 并且由于键长的差异, 配位多面 体可能会更加扭曲，这有利于大的倍频响应. 该工作 为探索卤素硫属化合物中红外非线性光学材料提供了 全景图和行动指南.

\subsection{4 含有 $\mathrm{d}^{0}$ 过渡族阳离子和 $\mathrm{O} / \mathrm{F}$ 杂化功能基团的 非线性光学材料}

潘世烈和杨志华课题组 ${ }^{[68]}$ 用第一性原理方法系 统调研了含 $\mathrm{d}^{0}$ 过渡金属的 $\mathrm{O} / \mathrm{F}$ 混合配位功能基团 $\left(\mathrm{d}^{0}\right.$ $\mathrm{TMOFs}$ )的中红外非线性光学晶体材料. 探索了这类氟 化功能基团对带隙和倍频响应的影响机制. 研究发现, $\mathrm{d}^{0}$ 过渡金属的氟化功能基团有利于产生较大的带隙. 他们研究了 39 个非中心对称的 $\mathrm{d}^{0}$ 过渡金属氟氧化物. 着重计算和分析了 9 个碱金属/碱土金属 $\mathrm{d}^{0}$ 过渡金属氟 氧化物的非线性光学性能. 计算表明, 它们具有宽的带 隙 $(>3.0 \mathrm{eV})$ 和大的双折射 $(>0.07)$, 其中 2 例分别含 $\mathrm{W}$ 和 $\mathrm{Mo}$ 的氟氧化物也呈现了较强的倍频效应. 此外, 他们 对比分析了其他 $\mathrm{d}^{0} \mathrm{TMOFs}$ 功能基团, 发现在基本构筑 基元中引入氟离子有利于光学性能的提升. 由此说明, $\mathrm{d}^{0}$ 过渡金属氟氧化物可以作为探索新型中红外非线性 光学材料的潜在体系.

\section{3 第一性原理高通量笁选流水线系统(FHSP- NLO)及其应用}

如前文所述，第一性原理计算方法在发现非线 性光学材料方面取得了广泛的应用, 有很多成功的 例子. 尤其在深紫外氟代硼酸盐体系, 在理论研究提 出新的功能基团和材料设计策略之后，大量的实验 跟进合成了众多性能优异的新材料. 在这一过程中, 理论计算和实验相互促进, 形成了一套良性互动的 材料发现新模式. 众多的成功案例证明, 通过理论计 算在已知材料中发掘新的材料并在此基础上通过分 析提出新的功能基团和材料设计策略是一种行之有 效的方法, 值得推广到更多的材料体系. 受限于计算 方法, 上述单个研究所涉及的材料不超过 50 个, 对于 拥有更多数量的材料体系，人工处理很难完成大量 


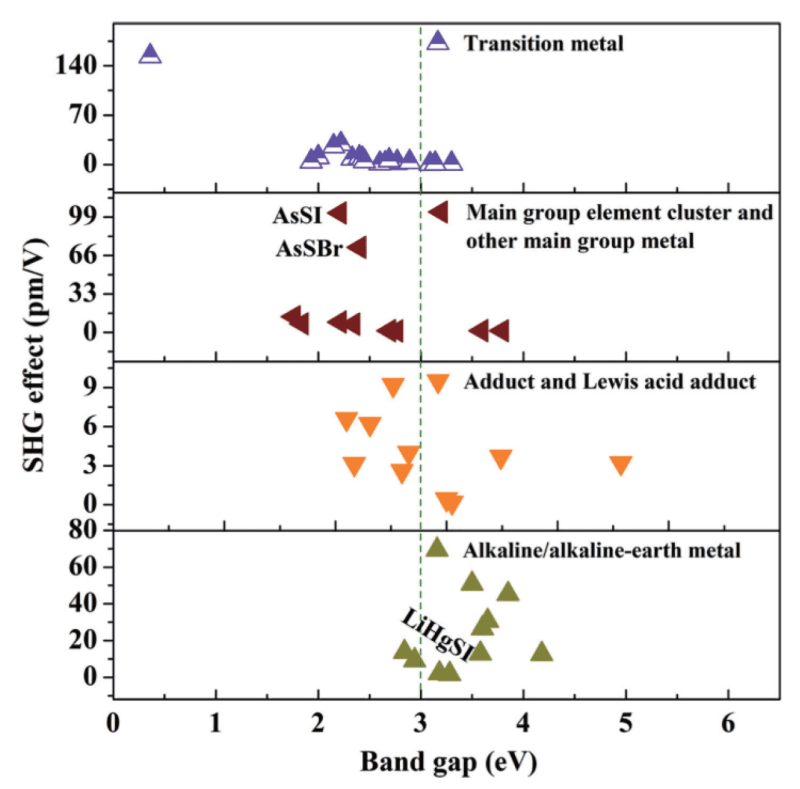

图 5 卤素硫属化物材料的能带和倍频系数分布 ${ }^{[67]}$ (网络版 彩图)

Figure 5 The distributions of all the mentioned chalcohalides in terms of band gaps and calculated the largest NLO coefficients in effective coefficients in this work [67] (color online).

的计算和分析. 需要可以实现自动化、批量化计算 和分析的工具, 对成千上万的已知材料进行地毯式 的搜索.

\subsection{FHSP-NLO简介及测试}

如图6所示, FHSP-NLO唯一需要的输入是晶体结 构文件. 之后该系统可以自动运行, 一次性计算和分析 成百上千个材料. 我们可以从无机晶体数据库(ICSD) 中根据元素组成搜索和下载具有非中心对称(NCS)结 构的晶体结构CIF文件. 在下载的结构中, 可能会包含 原子位置无序, 不同种类原子共占位, $\mathrm{H}$ 原子位置未定 的结构, 以及CIF文件格式错误的结构. 这些结构无法 被正确地计算，需要通过执行CIF检查代码加以排除. 然后使用cif2cell程序将CIF文件转换为.cell格式的文 件作为 $\mathrm{CASTEP}^{[69]}$ 的输入文件.

采用DFT、平面波基组和噟势方法的CASTEP程 序执行电子结构的计算. 根据以往大量的计算案例, 选 择模守恒噟势 (NCP) 和广义梯度近似(GGA)下的 Perdew-Burke-Ernzerhof $(\mathrm{PBE})^{[70]}$ 交换-关联泛函. 自洽 场计算的能量收玫标准为 $1 \times 10^{-6} \mathrm{eV}$. 自洽场计算的 Monkhorst-Pack $k$ 点取样间距设置为 $0.071 / \AA$, 光学计

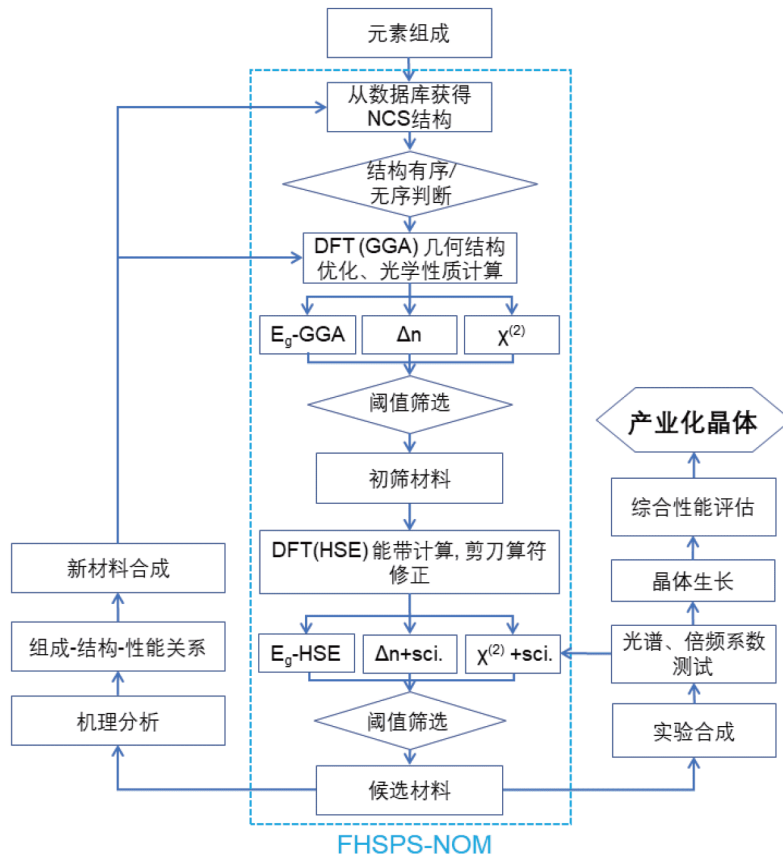

图 6 非线性光学材料第一性原理高通量篮选流程图(网络 版彩图)

Figure 6 The flow chart of the first-principles high-throughput screening pipeline for nonlinear optical materials (FHSP-NLO) (color online).

算的 $k$ 点间隔为 $0.041 / \AA$. 为确保折射率和倍频系数的 收玫, 空带数设置为价带的 3 倍.

完成光学计算后, 根据其电子结构计算其 $E_{\mathrm{g}} 、 \Delta n$ 和晶体的倍频系数 $\chi^{(2)}$. OptaDOS ${ }^{[71,72]}$ 代码用于计算折 射率. 用由Sipe等提出并由李明宪等开发的程序计算 倍频系数张量 $\chi^{(2)[20,73]}$. 计算公式如下:

$\chi_{\alpha \beta \gamma}{ }^{(2)}=\chi_{\alpha \beta \gamma}{ }^{(2)}(\mathrm{VE})+\chi_{\alpha \beta \gamma}{ }^{(2)}(\mathrm{VH})$

$\chi_{\alpha \beta \gamma}{ }^{(2)}(\mathrm{VE})=$

$\frac{e^{3}}{2 \hbar^{2} m^{3}} \sum_{v c c} \int \frac{\mathrm{d}^{3} k}{4 \pi^{3}} P(\alpha \beta \gamma) \operatorname{Im}\left[P_{c v^{\alpha}}^{\alpha} P_{c c^{\prime}}^{\beta} P_{c v}^{\gamma}\right]\left(\frac{1}{\omega_{c v}^{3} \omega_{v c^{\prime}}^{2}}+\frac{2}{\omega_{v c}^{4} \omega_{c^{\prime} v}}\right)$

$\chi_{\alpha \beta \gamma}{ }^{(2)}(\mathrm{VH})=$

$\frac{e^{3}}{2 \hbar^{2} m^{3}} \sum_{v v^{\prime} c} \int_{\frac{\mathrm{d}^{3} k}{4 \pi^{3}}} P(\alpha \beta \gamma) \operatorname{Im}\left[P_{v v^{\prime}}^{\alpha} P_{c v^{\beta}}^{\beta} P_{c v}^{\gamma}\right]\left(\frac{1}{\omega_{c v^{3} \omega_{v^{\prime} c}^{2}}^{2}}+\frac{2}{\omega_{v c}^{4} \omega_{c v^{\prime}}}\right)$

根据计算得到的性能，将满足性能指标的晶体篎 选出来作为初篮材料. 通常来说, 用 GGA交换-关联泛 函计算的带隙比实验值偏低. 因此导致计算的倍频系 数与双折射被不同程度地高估. 为了提高计算精度, 
我们采用在GPU上运行的平面波程序PWmat计算HSE 杂化泛函带隙. 用 $E_{\mathrm{g}}$-HSE和 $E_{\mathrm{g}}$-GGA之差作为剪刀算 符修正 $\Delta n$ 和 $\chi^{(2)}$. 最后, 从初篮材料中挑选出满足标准 的材料作为候选材料.

随后，合成候选材料的多晶样品、测量带隙和倍 频响应. 如果实验测量结果与预期结果一致, 可以生 长大尺寸的单晶以进行全面测试以及应用评估. 另一 方面，大量的计算数据和性能优异的目标材料可以用 来做统计学和结构-性能关系研究, 找到影响材料性能 的组份-结构“关键基因”, 进一步指导合成具有优异非 线性光学性能的新材料.

为了测试该方法的精度和可行性, 我们首先测试 了 12 个知名的非线性光学材料, 这些材料应用于深紫 外、紫外、可见-近红外和中/远红外的不同波段，包 括KBBF、BBO、LBO、CBO、CLBO、KTP、LN、 $\mathrm{BaAlBO}_{3} \mathrm{~F}_{2} 、 \mathrm{SrB}_{4} \mathrm{O}_{7} 、 \mathrm{Li}_{2} \mathrm{~B}_{4} \mathrm{O}_{7} 、 \mathrm{BiB}_{3} \mathrm{O}_{6}\left(\mathrm{C}_{2}\right) 、 \mathrm{Ag}-$ $\mathrm{GaS}_{2} 、 \mathrm{AgGaSe}_{2}$ 和 $\mathrm{ZnGeP}_{2}$. 如图7所示, 对角线代表预 测值和实验值完全相等的位置. 图中的正方形分布在 对角线附近，标志着预测值和实验值很接近. 计算的 $E_{\mathrm{g}} 、 \Delta n$ 和 $\chi^{(2)}$ 的平均绝对误差分别为 $18.3 \% 、 29.4 \%$ 和 $27.4 \%$. 结果表明, 我们使用的方法正确性高, 可靠地预 测了非线性光学的相关性能.

\subsection{FHSP-NLO 在硼酸盐体系的应用}

接下来, 我们对砋酸盐体系做了研究, 硼酸盐是搜 寻紫外/深紫外非线性光学材料的首选体系, 其中的很 多材料都被研究过. 我们搜索的规则是必须包括 $\mathrm{B}$ 和 $\mathrm{O}$
元素，排除镧系元素、钣系元素、过渡金属以确保材 料具有较大的带隙, 此外 $\mathrm{C}$ 元素也被排除在外以避免 有机-无机杂化晶体. 最后有 289 个晶体结构计算成功. 所有的计算是在一台 220 个 CPU的计算机集群上进行 的，总共花了 3 天时间，平均每个晶体只用了 5.5 个 CPU 核/小时, 这说明这种方法非常高效.

我们将计算得到的 $E_{\mathrm{g}}$-GGA、 $\Delta n$ 和 $\chi^{(2)}$ 结果两两 组合作图，可以发现这三者之间有趣的统计规律率. 如图 $8 \mathrm{a}, \mathrm{b}$ 所示，随着带隙的增大，硼酸盐的倍频系数 和双折射呈明显减小的趋势．相反，在 $\Delta n$ 和 $\chi^{(2)}$ 之间 没有明显的关系. 我们所有选定的材料均为氟代硼 酸氟或硼酸铍氟化物，包括 $\mathrm{CaB}_{5} \mathrm{O}_{7} \mathrm{~F}_{3} 、 \mathrm{SrB}_{5} \mathrm{O}_{7} \mathrm{~F}_{3}$ 、 $\mathrm{Li}_{2} \mathrm{~F}_{2} \mathrm{~B}_{6} \mathrm{O}_{9} 、 \mathrm{CsKB}_{8} \mathrm{O}_{12} \mathrm{~F}_{2} 、 \mathrm{NH}_{4} \mathrm{~B}_{4} \mathrm{O}_{6} \mathrm{~F} 、 \mathrm{RbB}_{4} \mathrm{O}_{6} \mathrm{~F}$ 、 $\mathrm{KBe}_{2} \mathrm{BO}_{3} \mathrm{~F}_{2} 、 \mathrm{RbBe}_{2} \mathrm{BO}_{3} \mathrm{~F}_{2}$ 和 $\mathrm{NaBe}_{2} \mathrm{BO}_{3} \mathrm{~F}_{2}$. 氟代嗍酸 盐打破典型硼酸盐的统计规律, 实现了三种性能之间 的平衡. 此外, 我们还发现了 5 个新的非线性光学晶 体, 即 $\mathrm{B}_{2} \mathrm{~S}_{2} \mathrm{O}_{9}{ }^{[74]} 、 \mathrm{Al}_{4} \mathrm{~B}_{6} \mathrm{O}_{15}{ }^{[75]} 、 \mathrm{HPNa}_{2} \mathrm{~B}_{4} \mathrm{O}_{7}{ }^{[76]}$ 、 $\mathrm{KB}\left(\mathrm{SO}_{3} \mathrm{Cl}\right)_{4}{ }^{[77,78]}$ 和 $\mathrm{H}_{3} \mathrm{BO}_{3}-3 \mathrm{~T}^{[79]}$. 除此之外, 还有两种水 合嶰酸盐 $\left[\mathrm{Ca}_{2} \mathrm{~B}_{5} \mathrm{O}_{9}\right] \cdot\left[\mathrm{H}(\mathrm{OH})_{2}\right]^{[80]}$ 和 $\mathrm{Ca}\left(\mathrm{B}_{8} \mathrm{O}_{11}(\mathrm{OH})_{4}{ }^{[81]}\right.$ 被 $^{\circ}$ 确定为有潜力的非线性光学材料. 以上工作表明, FHSP-NLO是一个强大而有效的工具, 可以从数据库 中笁选出新的优异非线性光学晶体材料.

\subsection{FHSP-NLO在锗酸盐体系的应用}

锗酸盐可实现 $3 \sim 5 \mu \mathrm{m}$ 的透过. 例如，最近报道的 $\mathrm{AI}_{4} \mathrm{Li}_{2} \mathrm{TiOGe}_{4} \mathrm{O}_{12} \quad(\mathrm{AI}=\mathrm{Rb}, \mathrm{K})^{[82,83]}$ 可覆盖0.28 5.6 $\mu \mathrm{m}$ 的透过范围. 我们检索了 ICSD数据库, 获得了 519 个含
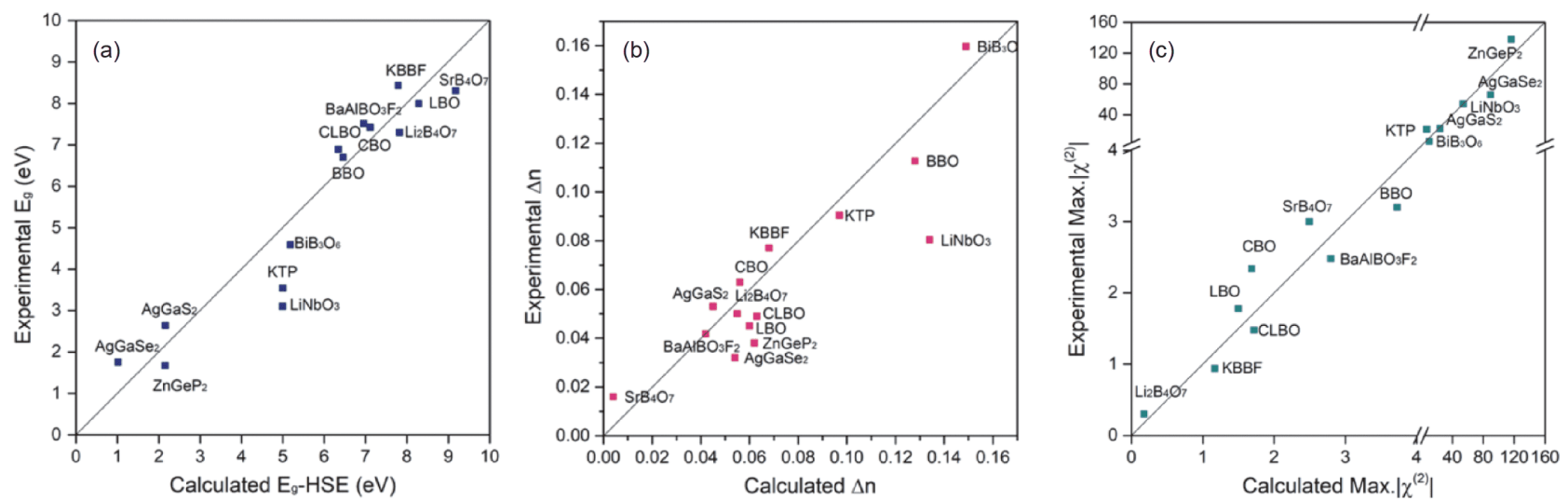

图 712 种知名非线性光学晶体的带隙 $E_{\mathrm{g}}$ (a)、双折射 $\Delta n(\mathrm{~b})$ 、倍频系数 $\chi^{(2)}$ (c) 的计算值和实验值对比 ${ }^{[27]}$ (网络版彩图)

Figure 7 Comparison of calculated and experimental values of 12 well-known NLO materials, including $E_{\mathrm{g}}$-HSE $v s$. measured band gap (a), birefringence $\Delta n$ (b), and maximum SHG coefficients Max. $\left|\chi^{(2)}\right|$ (c) [27] (color online). 

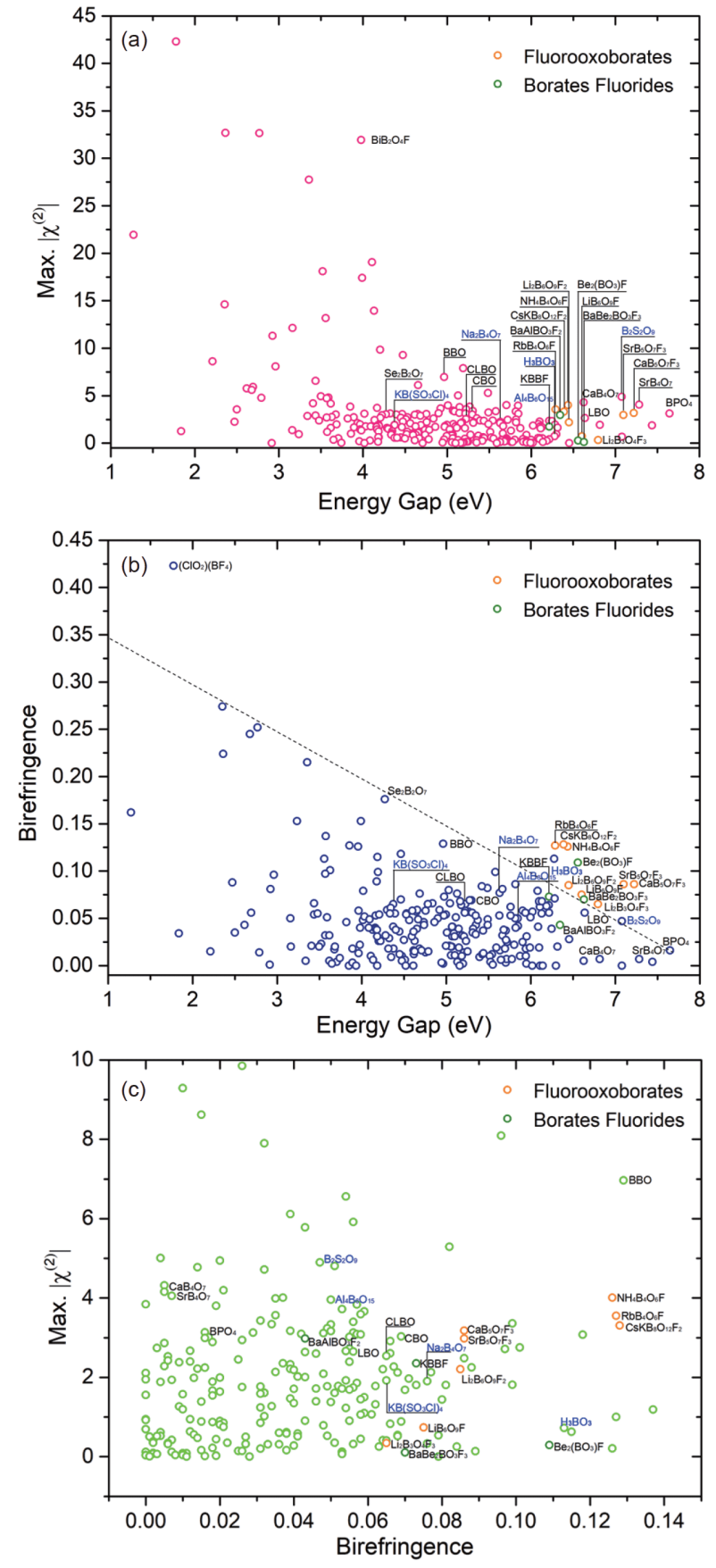

图 8 计算结果的统计图. (a) 能带vs. 最大倍频系数; (b) 能 带vs. 双折射; (c) 双折射 vs. 最大倍频系数 ${ }^{[27]}$ (网络版彩图)

Figure 8 The statistical chart of calculated results for almost three hundred borates. (a) Band gap vs. birefringence; (b) band gap vs. SHG coefficients; (c) birefringence vs. SHG coefficients [27] (color online).

有 $\mathrm{Ge}$ 和O元素的非中心对称晶体结构, 其中 228 个无序 结构(包含原子位置无序和固溶体), 26 个结构的 $\mathrm{H}$ 原子
未定，68个高压下解析的结构. 剩余197个有序、所有 原子位置都定下来的结构, 排除掉含有未满 $\mathrm{d}$ 或 $\mathrm{f}$ 电子 的阳离子, 剩余144个结构. 用FHSP-NLO系统计算了 这144个锗酸盐晶体, 最终 128 个结构计算成功, 获得了 材料的带隙 $E_{\mathrm{g}}-\mathrm{GGA}$ 、倍频系数 $\chi^{(2)}$ 和双折射 $\Delta n$. 如图9 所示, 其中 6 个锗酸盐倍频系数大于 $8 \mathrm{pm} / \mathrm{V}\left(10 \times \chi_{36}\right.$ (KDP)). 我们使用HSE杂化泛函更精确地预测了这6个 材料的带隙, 并且用剪刀算符修正了它们的倍频系数 和双折射. 修正后, 我们获得了 3 个满足性能指标的锗 酸盐材料 $\mathrm{Ba}_{2} \mathrm{TiGe}_{2} \mathrm{O}_{8} 、 \mathrm{~Pb}_{3} \mathrm{Ga}_{2} \mathrm{Ge}_{4} \mathrm{O}_{14} 、 \mathrm{~Pb}_{3} \mathrm{O}\left(\mathrm{GeO}_{4}\right)$. 随 后, 我们合成了这三种材料的粉末样品. 利用KurtzPerry法测试表明, $\mathrm{Ba}_{2} \mathrm{TiGe}_{2} \mathrm{O}_{8}$ 和 $\mathrm{Pb}_{3} \mathrm{Ga}_{2} \mathrm{Ge}_{4} \mathrm{O}_{14}$ 具有非 常大的粉末倍频效应, 分别为 1.2 倍和 0.8 倍 $\mathrm{AgGaS}_{2}$. $\mathrm{Pb}_{3} \mathrm{O}\left(\mathrm{GeO}_{4}\right)$ 很可能是无序结构, 其有序结构的解析有 一定的不合理性，这也导致了对其倍频效应预测的偏 离 $^{[84]}$.

\subsection{FHSP-NLO 在氮代硅酸盐体系的应用}

硅酸盐具有丰富的结构、高的激光损伤阈值, 容 易生长大尺寸单晶, 本应是寻找紫外非线性光学材料 的优势体系，但是其过小的双折射阻碍了相位匹配. 在前期研究的启发下, 我们用FHSP-NLO方法发现氮 代硅酸盐可以克服硅酸盐双折射过小的不足, 是一类 潜在的紫外非线性光学材料. 氮代硅酸盐中, 氮原子 可以部分取代氧原子形成 $\mathrm{SiN}_{x} \mathrm{O}_{4-x} \quad(x=2,3)$ 混合阴离 子四面体. 我们首先用Gussian的DFT方法计算了 $\left(\mathrm{SiN}_{x} \mathrm{O}_{4-x}\right)^{(x+4)-}(x=1,2,3)$ 阴离子基团的电子结构和光 学性质. 结果表明极性 $\left(\mathrm{SiNO}_{3}\right)^{5-}$ 、 $\left(\mathrm{SiN}_{2} \mathrm{O}_{2}\right)^{6-}$ 和 $\left(\mathrm{SiN}_{3}-\right.$ $\mathrm{O})^{7-}$ 基团表现出明显的极化率各向异性，分别为5.2、 9.0和4.8. 为了清楚地展示它们的极化率各向异性, 我 们画了极化率各向异性曲面(图10). 值得注意的是, $\mathrm{O}$ 被N取代显著减小了HOMO-LUMO间隙. 但是在氮代 硅酸盐晶体中, $\mathrm{N}$ 原子与一个以上的 $\mathrm{Si}$ 原子配位, 这将 消除 $\mathrm{N}$ 上的悬挂键, 以扩大带隙实现紫外透过.

随后, 我们在ICSD数据库中找到九个非中心对称 的氮代硅酸盐. 用FHSP-NLO篮选出 $\mathrm{Si}_{2} \mathrm{~N}_{2} \mathrm{O}$ 和 LiSiON (晶体结构如图11所示)具有宽带隙 $(6.49$ 和6.95 eV)、 大的双折射(在 $1064 \mathrm{~nm}$ 处为 0.102 和 0.060 ), 以及较大 的倍频系数 $\left(3.3\right.$ 和 2.2 倍的 $\left.d_{36}(\mathrm{KDP})\right)$. 通过研究分波态 密度, 我们发现 $\mathrm{N}$ 原子与 $\mathrm{Si}$ 原子三配位可以尽可能消 除 $\mathrm{N}$ 的悬挂键, 以增加带隙. 这项研究提供了一种设计 
(i)
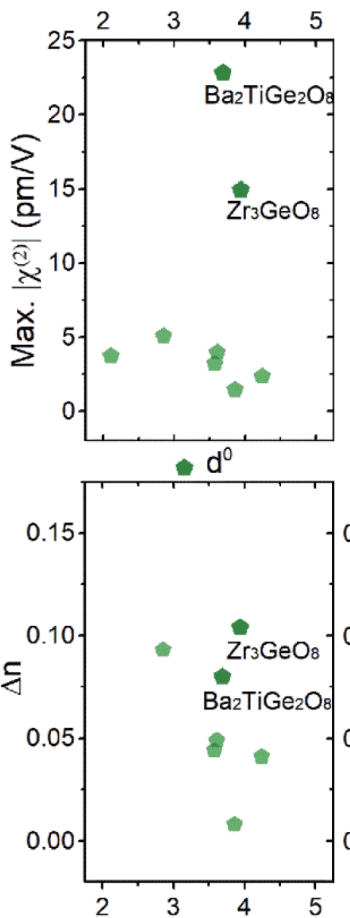

(ii)

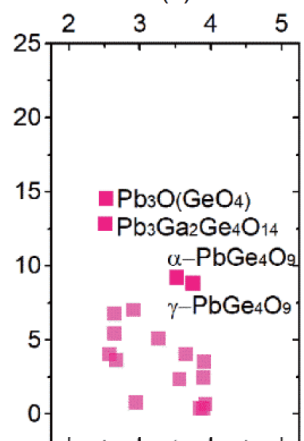

- Lone Pair

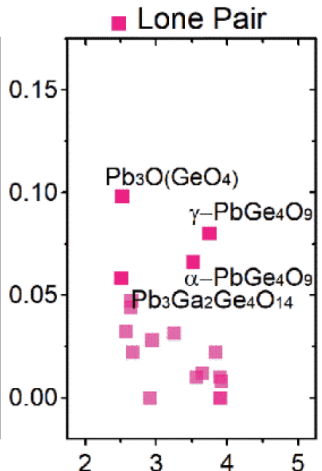

(iii)
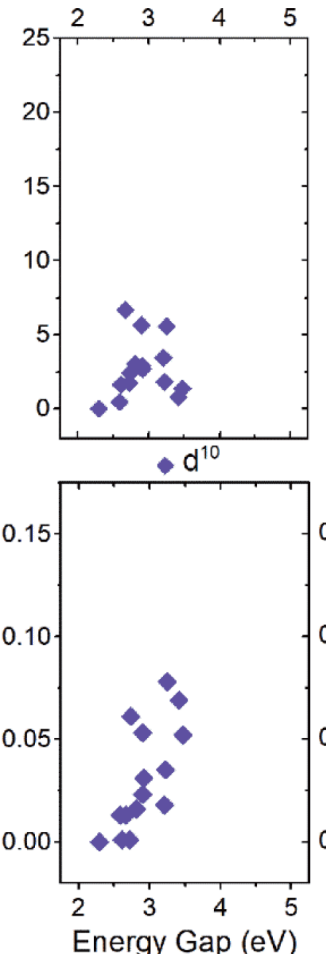

(iv)

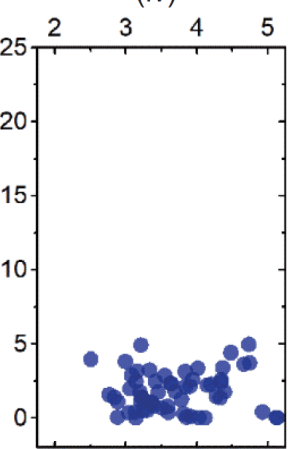

- IA, IIA, Y, Sc, La, Lu

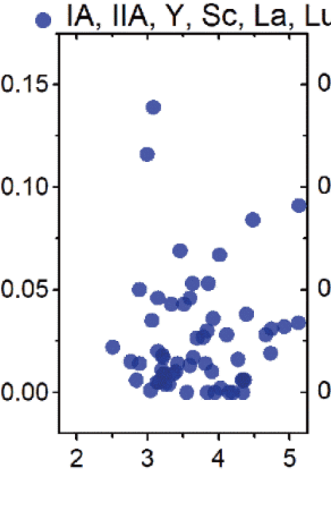

(v)

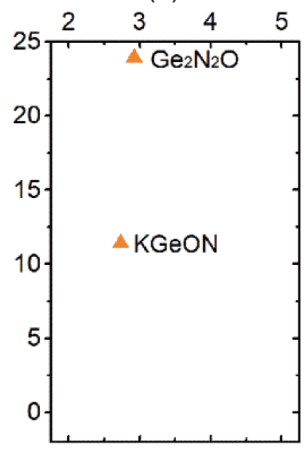

Oxynitride

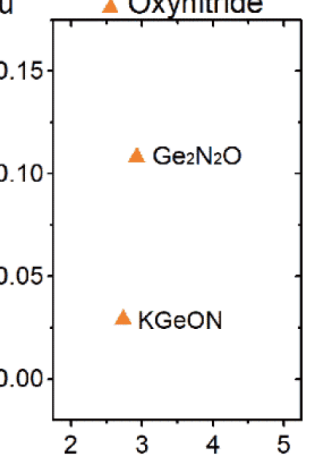

图 9 非中心对称锗酸盐晶体材料的带隙 $\left(E_{\mathrm{g}}\right)$ 、最大倍频系数 $\left(\operatorname{Max} . \chi^{(2)} \mid\right)$ 、双折射 $(\Delta n)$ 的分布. 按照所含阳离子分为: (i) $\mathrm{d}^{0}-$ 过 渡族金属; (ii) 孤对电子阳离子; (iii) $\mathrm{d}^{10}$-过渡族金属; (iv) 碱金属、碱土金属、 $\mathrm{Y}^{3+} 、 \mathrm{Sc}^{3+} 、 \mathrm{La}^{3+} 、 \mathrm{Lu}^{3+} ;(\mathrm{v})$ 氮化硅酸盐 ${ }^{[84]}$ (网络 版彩图)

Figure 9 The calculated maximum $\chi^{(2)}$ values and birefringence $(\Delta n)$ dispersion $v$ s. band gap calculated by using GGA functionals of selected germanates that are divided into five categories, i.e., geminates containing: (i) $\mathrm{d}^{0}$-transition metal cations; (ii) lone-pair activity cations; (iii) $\mathrm{d}^{10}$ transition metal cations; (iv) alkali and alkaline-earth metal cations, $\mathrm{Y}^{3+}, \mathrm{Sc}^{3+}, \mathrm{La}^{3+}, \mathrm{Lu}^{3+}$ are also including in this category; (v) germanium oxynitride [84] (color online).

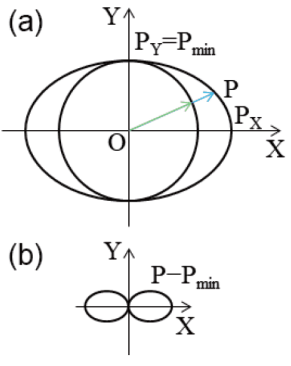

(c)
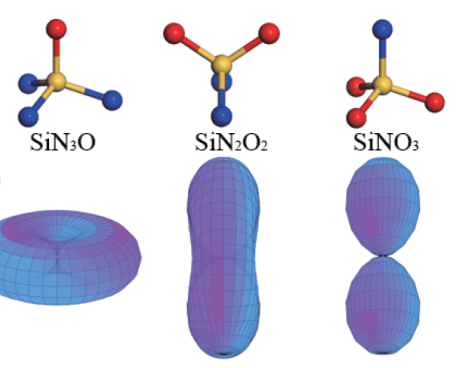

图 10 (a) 平面各项异性极化率示意图; (b) 极化率各向异性 曲线; (c) $\left(\mathrm{SiN}_{3} \mathrm{O}\right)^{7-} 、\left(\mathrm{SiN}_{2} \mathrm{O}_{2}\right)^{6-}$ 和 $\left(\mathrm{SiNO}_{3}\right)^{5-}$ 基团; (d) 三个基 团对应的极化率各向异性曲面 ${ }^{[85]}$ (网络版彩图)

Figure 10 (a) The two-dimension diagram of the anisotropic polarizability and (b) the corresponding polarizability anisotropy curve (surface if three-dimension) that is defined as the difference between the polarizability ellipsoid and the sphere with a radius determined by the minimum polarizability at same direction. (c) The representation of $\left(\mathrm{SiN}_{3} \mathrm{O}\right)^{7-},\left(\mathrm{SiN}_{2} \mathrm{O}_{2}\right)^{6-}$, and $\left(\mathrm{SiNO}_{3}\right)^{5-}$ groups and (d) their polarizability anisotropy surfaces [85] (color online).
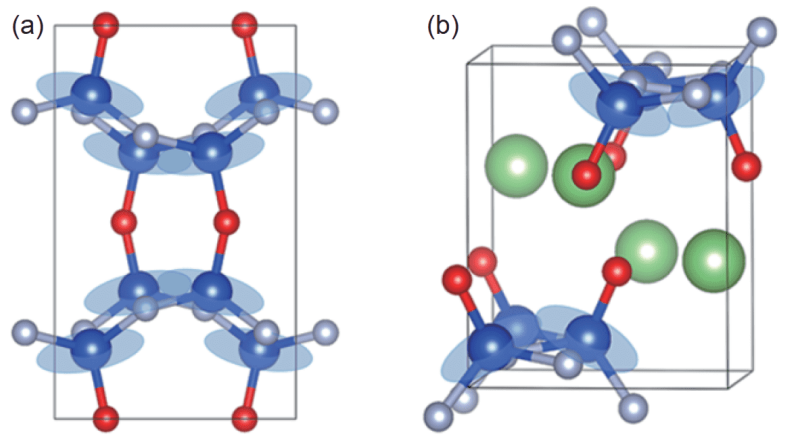

图 $11 \mathrm{Si}_{2} \mathrm{~N}_{2} \mathrm{O}$ (a)和LiSiON (b)的晶体结构示意图. 通过棍 棒连接的蓝、红、灰、绿颜色的球体分别表示 $\mathrm{Si} 、 \mathrm{O} 、 \mathrm{~N}$ 、

$\mathrm{Li}$ 原子. 环形平面表示 $\mathrm{SiON}_{3}$ 四面体基团的最大极化平面(网 络版彩图)

Figure 11 The crystal structures of $\mathrm{Si}_{2} \mathrm{~N}_{2} \mathrm{O}$ (a) and $\mathrm{LiSiON}$ (b). The blue, red, grey, and green balls connected by sticks are $\mathrm{Si}, \mathrm{O}, \mathrm{N}$, and $\mathrm{Li}$ atoms, respectively. The circle planes represent the maximum polarizability plane of $\mathrm{SiON}_{3}$ tetrahedra (color online). 
和发现紫外非线性光学晶体的新策略和新体系 ${ }^{[85]}$.

\section{4 总结与展望}

在非线性光学晶体领域, 从早期的“阴离子基团理 论”, 到现在第一性原理计算方法普及, 理论计算和实 验紧密结合, 相互促进, 发现了一系列性能优异的非线 性光学材料, 也使得我国一直处于该领域的世界前列. 第一性原理方法在深紫外氟代嗍酸盐体系、氟代磷酸
盐体系、氰尿酸盐体系的发展过程中起到了重要的推 动作用. 非线性光学材料领域的发展, 生动展现了理论 研究在材料发现中的重要作用. 在众多材料研究领域, 非线性光学材料的发展是理论结合实验的典型代表和 成功案例. 第一性原理高通量篮选流水线系统(FHSPNLO)整合了多种计算和分析软件, 可实现大规模的材 料篮选. 随着该系统的应用, 更多的潜在的体系、性能 优异的材料、新颖的功能基团将会被发掘出来. 这将 显著加速非线性光学材料的研究步伐.

致谢感谢中国科学院新疆理化技术研究所潘世烈和杨志华研究员、台湾淡江大学李明宪教授、山东大学王继扬 和于浩海教授、新疆大学井群和陈兆慧教授、天津理工大学俞洪伟和吴红萍教授的讨论与合作. 感谢与我合作的史国 强博士、韩国鹏博士、陈幸龙博士。感谢审稿专家提出修改意见和建议, 在此一并致谢.

\section{参考文献}

1 Franken PA, Hill AE, Peters CW, Weinreich G. Phys Rev Lett, 1961, 7: 118-119

2 Chen C, Sasaki T, Li R, Wu Y, Lin Z, Mori Y, Hu Z, Wang J, Uda S, Yoshimura M, Kaneda Y. Nonlinear Optical Borate Crystals. Weinheim: Wiley-VCH Verlag GmbH \& Co. KGaA, 2012

3 Wu K, Pan S. Coord Chem Rev, 2018, 377: 191-208

4 Wu C, Yang G, Humphrey MG, Zhang C. Coord Chem Rev, 2018, 375: 459-488

5 Savage N. Nat Photon, 2007, 1: 83-85

6 Xia Y, Chen C, Tang D, Wu B. Adv Mater, 1995, 7: 79-81

7 Lin Z, Jiang X, Kang L, Gong P, Luo S, Lee MH. J Phys D-Appl Phys, 2014, 47: 253001

8 Mutailipu M, Pan S. Angew Chem, 2020, 132: 20480-20496

9 Tran TT, Yu H, Rondinelli JM, Poeppelmeier KR, Halasyamani PS. Chem Mater, 2016, 28: 5238-5258

10 Liang F, Kang L, Lin Z, Wu Y, Chen C. Coord Chem Rev, 2017, 333: 57-70

11 Smith WL. Appl Opt, 1977, 16: 1798

12 Boyd GD, Miller RC, Nassau K, Bond WL, Savage A. Appl Phys Lett, 1964, 5: 234-236

13 Stolzenberger RA. Appl Opt, 1988, 27: 3883-3886

14 Chen C, Wang Y, Xia Y, Wu B, Tang D, Wu K, Wenrong Z, Yu L, Mei L. J Appl Phys, 1995, 77: 2268-2272

Chen C, Wu Y, Jiang A, Wu B, You G, Li R, Lin S. J Opt Soc Am B, 1989, 6: 616-621

Wu Y, Sasaki T, Nakai S, Yokotani A, Tang H, Chen C. Appl Phys Lett, 1993, 62: 2614-2615

1 Sasaki T, Mori Y, Yoshimura M. Optical Mater, 2003, 23: 343-351

Ohmer MC, Pandey R. MRS Bull, 1998, 23: 16-22

Chen C, Ye N, Lin J, Jiang J, Zeng W, Wu B. Adv Mater, 1999, 11: 1071-1078

Lin J, Lee MH, Liu ZP, Chen C, Pickard CJ. Phys Rev B, 1999, 60: 13380-13389

He R, Huang H, Kang L, Yao W, Jiang X, Lin Z, Qin J, Chen C. Appl Phys Lett, 2013, 102: 231904

Jiang X, Zhao S, Lin Z, Luo J, Bristowe PD, Guan X, Chen C. J Mater Chem C, 2014, 2: 530-537

Lei BH, Yang Z, Pan S. Chem Commun, 2017, 53: 2818-2821

Lei BH, Yang Z, Yu H, Cao C, Li Z, Hu C, Poeppelmeier KR, Pan S. J Am Chem Soc, 2018, 140: 10726-10733

Yang Z, Lei BH, Zhang W, Pan S. Chem Mater, 2019, 31: 2807-2813

Wu H, Yu H, Yang Z, Hou X, Su X, Pan S, Poeppelmeier KR, Rondinelli JM. J Am Chem Soc, 2013, 135: 4215-4218

Zhang B, Zhang X, Yu J, Wang Y, Wu K, Lee MH. Chem Mater, 2020, 32: 6772-6779 
Becker P. Adv Mater, 1998, 10: 979-992

Zhao S, Gong P, Luo S, Bai L, Lin Z, Tang Y, Zhou Y, Hong M, Luo J. Angew Chem Int Ed, 2015, 54: 4217-4221

Sun CF, Hu CL, Xu X, Yang BP, Mao JG. J Am Chem Soc, 2011, 133: 5561-5572

Chen C, Wang Y, Wu B, Wu K, Zeng W, Yu L. Nature, 1995, 373: 322-324

Pilz T, Jansen M. Z anorg allg Chem, 2011, 637: 2148-2152

Pilz T, Nuss H, Jansen M. J Solid State Chem, 2012, 186: 104-108

Cakmak G, Nuss J, Jansen M. Z anorg allg Chem, 2009, 635: 631-636

Zhang B, Shi G, Yang Z, Zhang F, Pan S. Angew Chem Int Ed, 2017, 56: 3916-3919

Shi G, Wang Y, Zhang F, Zhang B, Yang Z, Hou X, Pan S, Poeppelmeier KR. J Am Chem Soc, 2017, 139: 10645-10648

Zhang Z, Wang Y, Zhang B, Yang Z, Pan S. Angew Chem Int Ed, 2018, 57: 6577-6581

Wang Y, Zhang B, Yang Z, Pan S. Angew Chem Int Ed, 2018, 57: 2150-2154

Wang X, Wang Y, Zhang B, Zhang F, Yang Z, Pan S. Angew Chem Int Ed, 2017, 56: 14119-14123

Luo M, Liang F, Song Y, Zhao D, Xu F, Ye N, Lin Z. J Am Chem Soc, 2018, 140: 3884-3887

Zhang Z, Wang Y, Zhang B, Yang Z, Pan S. Inorg Chem, 2018, 57: 4820-4823

Mutailipu M, Zhang M, Zhang B, Wang L, Yang Z, Zhou X, Pan S. Angew Chem Int Ed, 2018, 57: 6095-6099

Xia M, Li F, Mutailipu M, Han S, Yang Z, Pan S. Angew Chem Int Ed, 2021, 60: 14650-14656

Zhang B, Han G, Wang Y, Chen X, Yang Z, Pan S. Chem Mater, 2018, 30: 5397-5403

Xiong L, Chen J, Lu J, Pan CY, Wu LM. Chem Mater, 2018, 30: 7823-7830

Lu J, Yue J-N, Xiong L, Zhang W-K, Chen L, Wu L-M. J Am Chem Soc, 2019, 141: 8093-8097

Kang L, Zhang X, Liang F, Lin Z, Huang B. Angew Chem Int Ed, 2019, 58: 10250-10254

Kang L, Liang F, Lin Z, Liu F, Huang B. Inorg Chem, 2018, 57: 15001-15008

Kang L, Liang F, Lin Z, Huang B. Inorg Chem, 2019, 58: 77-80

Kalmutzki M, Ströbele M, Wackenhut F, Meixner AJ, Meyer HJ. Angew Chem Int Ed, 2014, 53: 14260-14263

Kalmutzki M, Wang X, Meixner AJ, Meyer HJ. Cryst Res Tech, 2016, 51: 460-465

Kalmutzki M, Ströbele M, Wackenhut F, Meixner AJ, Meyer HJ. Inorg Chem, 2014, 53: 12540-12545

Kalmutzki M, Ströbele M, Meyer HJ. Dalton Trans, 2013, 42: 12934-12939

Liang F, Kang L, Zhang X, Lee MH, Lin Z, Wu Y. Cryst Growth Des, 2017, 17: 4015-4020

Liang F, Wang N, Liu X, Lin Z, Wu Y. Chem Commun, 2019, 55: 6257-6260

Xia M, Zhou M, Liang F, Meng X, Yao J, Lin Z, Li R. Inorg Chem, 2018, 57: 32-36

Meng X, Yin W, Xia M. Coord Chem Rev, 2021, 439: 213916

Lin D, Luo M, Lin C, Xu F, Ye N. J Am Chem Soc, 2019, 141: 3390-3394

Lu J, Lian YK, Xiong L, Wu QR, Zhao M, Shi KX, Chen L, Wu LM. J Am Chem Soc, 2019, 141: 16151-16159

Luo M, Lin C, Lin D, Ye N. Angew Chem Int Ed, 2020, 59: 15978-15981

Xia M, Mutailipu M, Li F, Yang Z, Pan S. Cryst Growth Des, 2021, 21: 1869-1877

Song Y, Luo M, Lin D, Lin C, Wang Z, Long X, Ye N. ACS Omega, 2021, 6: 9263-9268

Wen X, Lin C, Luo M, Fan H, Chen K, Ye N. Sci China Mater, 2021, 64: 2008-2016

Wu C, Jiang X, Wang Z, Sha H, Lin Z, Huang Z, Long X, Humphrey MG, Zhang C. Angew Chem Int Ed, 2021, 60: 14806-14810

Kang L, Zhou M, Yao J, Lin Z, Wu Y, Chen C. J Am Chem Soc, 2015, 137: 13049-13059

Li H, Li G, Wu K, Zhang B, Yang Z, Pan S. Chem Mater, 2018, 30: 7428-7432

Gao L, Huang J, Guo S, Yang Z, Pan S. Coord Chem Rev, 2020, 421: 213379

Huang J, Guo S, Zhang Z, Yang Z, Pan S. Sci China Mater, 2019, 62: 1798-1806

Clark SJ, Segall MD, Pickard CJ, Hasnip PJ, Probert MIJ, Refson K, Payne MC. Z für Kristallographie - Crystline Mater, 2005, 220: 567-570

Perdew JP, Burke K, Ernzerhof M. Phys Rev Lett, 1996, 77: 3865-3868

Nicholls RJ, Morris AJ, Pickard CJ, Yates JR. J Phys-Conf Ser, 2012, 371: 012062

Morris AJ, Nicholls RJ, Pickard CJ, Yates JR. Comput Phys Commun, 2014, 185: 1477-1485

Zhang B, Lee MH, Yang Z, Jing Q, Pan S, Zhang M, Wu H, Su X, Li CS. Appl Phys Lett, 2015, 106: 031906 


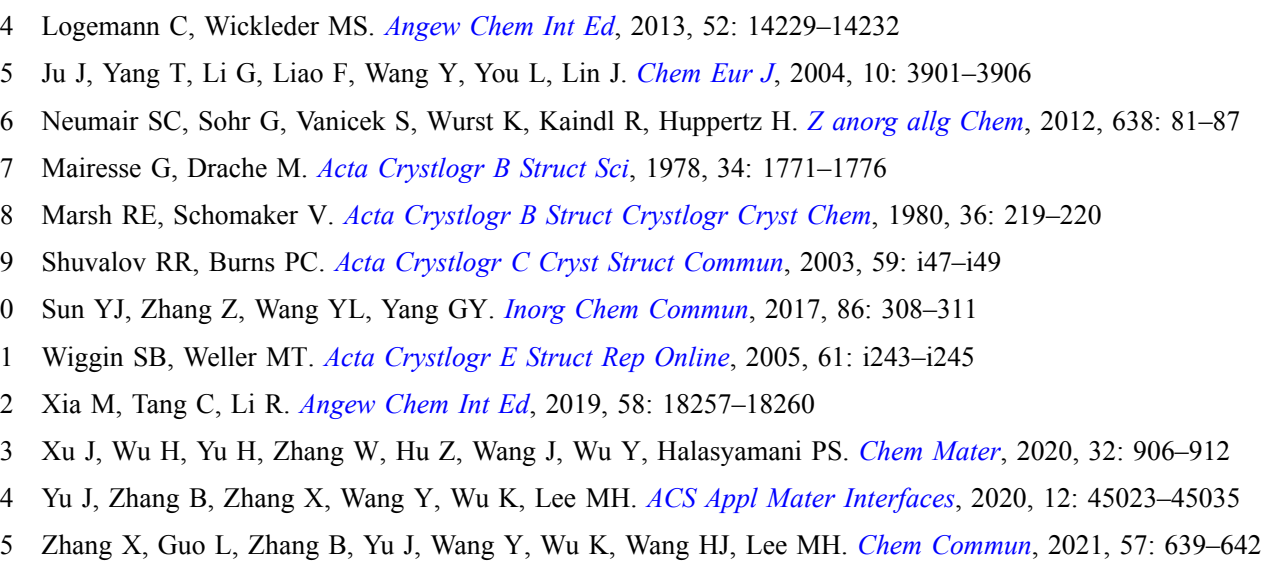

\title{
The application and development of the first principles calculation in nonlinear optical materials
}

\author{
Bingbing Zhang*, Kui Wu, Ying Wang, Daqing Yang \\ College of Chemistry and Environmental Science, Hebei University, Baoding 071002, China \\ *Corresponding author (email: zhangbb@hbu.edu.cn)
}

\begin{abstract}
Nonlinear optical (NLO) crystals need to meet multiple criteria, so it is a challenging and time-consuming task to find new NLO materials. The computational method that based on first-principles can screen out materials from a large number of materials by predicting their properties. In addition, the NLO-active groups of the materials can be identified by studying the "composition-structure-properties" relationship, which provides guidance for the synthesis of new materials. This paper summarizes the role and recent cases of computational materials in the discovery of new NLO materials. The first-principles high-throughput screening pipeline system for nonlinear optical materials (FHSP-NLO) is introduced emphatically. The system combines density functional theory (DFT) codes, linear and nonlinear optical property calculation codes, and data transformation and extraction codes, and can be automatically run to calculate and analyze a large number of materials at once. It has been successfully applied in systems including borates, germanates and nitride silicates, which provide a powerful and effective tool for searching new NLO materials.
\end{abstract}

Keywords: nonlinear optical crystals, second harmonic generation, first-principles, high-throughput

doi: $10.1360 /$ SSC-2021-0137 